\title{
Período de latência, progressão e duração do bloqueio da anestesia epidural com lidocaína, bupivacaína ou sua associação em cães
}

\author{
[Period of latency, progression and duration of blockade of epidural \\ anesthesia with lidocaine, bupivacaine or its association in dogs] \\ S.J. Ronchi ${ }^{1}$, M.I. Gehrcke ${ }^{2}$, D. Regalin ${ }^{3}$, N. Oleskovicz ${ }^{1}$ \\ ${ }^{1}$ Universidade do Estado de Santa Catarina - Lages, SC \\ ${ }^{2}$ Universidade Federal de Pelotas - Pelotas, RS \\ ${ }^{3}$ Universidade Federal de Jataí - Jataí, GO
}

\begin{abstract}
RESUMO
O objetivo deste estudo foi avaliar latência, duração do efeito, progressão cranial da lidocaína e da bupivacaína isoladas, ou em associação, pela via epidural, em cães, além de mensurar a pressão média do canal epidural antes e após a realização dela. Utilizaram-se 18 cães, alocados em três grupos, os quais receberam, por via epidural: lidocaína $2 \%$ (GL) $0,25 \mathrm{~mL} / \mathrm{kg}$; bupivacaína $0,5 \%$ (GB) no mesmo volume, ou a associação de ambas (GLB) na proporção de 1:1. Avaliaram-se as frequências cardíaca e respiratória e a pressão arterial sistólica (PAS) previamente aos tratamentos (M0) e até 60 minutos após a anestesia epidural. Ainda, avaliou-se a pressão no canal epidural antes e após a administração dos tratamentos, o período de latência, a progressão e a duração do bloqueio pelo pinçamento interdigital e do panículo paravertebral. Houve redução de $12 \%$ da PAS no GL em todos os momentos e de $16 \%$ aos 30 minutos no GLB quando comparados ao basal. A pressão média no espaço epidural antes e após a anestesia epidural foi de $-1,5( \pm 3,9)$ e $41( \pm 16) \mathrm{mmHg} ; 55 \%$ apresentaram pressão negativa no espaço epidural. O período

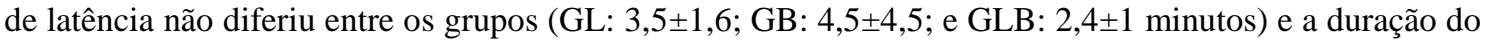
bloqueio foi maior no GB em relação ao GL (GL: 125 \pm 24 ; GB: $176 \pm 24$; e GLB: $153 \pm 35$ minutos). A progressão máxima dos anestésicos foi até L1-T13 no GL, L4-L3 no GB e L3-L2 no GLB. Conclui-se que a associação de lidocaína com bupivacaína não apresenta vantagens em relação ao uso dos fármacos isolados pela via epidural, tendo a lidocaína progredido mais cranialmente em relação à bupivacaína ou à associação. A lidocaína promoveu redução da PAS, mesmo quando associada à bupivacaína, permanecendo dentro dos valores de referência. Apenas 55\% dos cães apresentaram pressão média negativa no espaço epidural antes da administração dos fármacos, dessa forma o teste da gota pendente pode não ser eficiente para localização do espaço epidural em todos os animais.
\end{abstract}

Palavras-chave: anestesia espinhal, interação farmacológica, anestesia locorregional, anestésicos locais

\begin{abstract}
The objective of this study was to evaluate the latency, duration of the effect, and cranial progression of lidocaine and bupivacaine alone or in combination, by epidural route in dogs, and measuring the average pressure of the epidural channel before and after the completion thereof. Eighteen dogs were allocated in three groups, which received epidural: lidocaine 2\% (GL) $0.25 \mathrm{ml} / \mathrm{kg}$; bupivacaine $0.5 \%$ $(G B)$ in the same volume, or the association of both $(G L B)$ in a 1: 1 ratio. Heart and respiratory rates and systolic blood pressure (SBP) were evaluated before treatment $(M O)$ and up to 60 minutes after epidural anesthesia. In addition, the pressure in the epidural canal was evaluated before and after the administration of the treatments, latency period, progression and duration of the block by interdigital and paravertebral pannicus clamping. There was a 12\% decrease in SBP in the GL at all times and 16\% at 30 minutes in GLB when compared to the baseline. The mean pressure in the epidural space before and after epidural anesthesia was $-1.5( \pm 3.9)$ and $41( \pm 16) \mathrm{mmHg}), 55 \%$ presented negative pressure in the

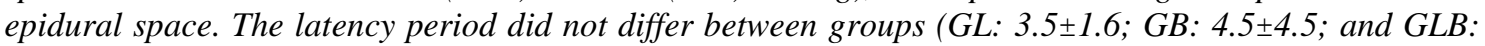

Recebido em 28 de agosto de 2017

Aceito em 7 de janeiro de 2019

E-mail: samuelronchi@yahoo.com.br 


\section{Ronchi et al.}

$2.4 \pm 1$ minutes) and the duration of blockade was higher in $G B(G L: 125 \pm 24, G B: 176 \pm 24$, and GLB: $153 \pm 35$ minutes). The maximum progression of anesthetics was up to L1-T13 in GL, L4-L3 in GB and L3L2 in GLB. It is concluded that the association of lidocaine with bupivacaine does not present advantages in relation to the use of the drugs isolated by the epidural route, with lidocaine progressing more cranially in relation to bupivacaine or the association. Lidocaine promoted the reduction of SBP, even when associated with bupivacaine, remaining within the reference values. Only 55\% of the dogs presented negative mean pressure in the epidural space before administration of the drugs, so the drop test may not be efficient for locating the epidural space in all animals.

Keywords: spinal anesthesia, pharmacological interaction, local anesthesia, local anesthetics

\section{INTRODUÇÃO}

A anestesia epidural apresenta diversos efeitos benéficos, entre eles proporciona uma analgesia adequada para procedimentos cirúrgicos, utilizando-se para essa finalidade muitas vezes os anestésicos locais (Bernards e Hill, 1992; Valverde, 2008). Entre os anestésicos locais, destaca-se a lidocaína e a bupivacaína, com características antagônicas que repercutem na sua utilização clínica. Do ponto de vista teórico, haveria tempos de latência e de duração menores com a lidocaína; já com a bupivacaína, ocorreriam efeitos clínicos contrários, latência e duração maiores (Butterworth e Strichartz, 1990; Bernards e Hill, 1992; Valverde, 2008). Tendo em vista isso, muitas vezes, na prática clínica, emprega-se a associação de ambos os fármacos visando a uma solução final com latência curta, característica da lidocaína, e duração prolongada, característica da bupivacaína.

No entanto, ao se observarem os dados descritos na literatura quanto aos efeitos da associação de lidocaína e bupivacaína, esses dados são conflitantes: em alguns estudos, a associação se demonstrou vantajosa (Cruz et al., 1997; Adetunji et al., 2001); já em outros, a associação não se mostrou superior quanto ao emprego dos fármacos isolados (Lawal e Adetunji, 2009). Além disso, em alguns desses estudos, os fármacos continham epinefrina em sua formulação, o que pode ter interferido nos resultados (Cruz et al., 1997; Adetunji et al., 2001). Ribotsky et al. (1996) são taxativos em afirmar que não há vantagens na associação de anestésicos locais devido à interferência nas características químicas entre os fármacos, não podendo prever o que acontecerá com a solução final; por consequência, não se pode prever o que acontecerá com a latência e duração da mistura de anestésicos locais.
Devido aos dados conflitantes apresentados na literatura e ao uso ainda regular da associação dos dois anestésicos na rotina hospitalar, o objetivo deste estudo foi avaliar o período de latência, a progressão cranial, a duração de bloqueio sensitivo da administração de lidocaína, bupivacaína ou sua associação por via epidural em cães submetidos à orquiectomia eletiva. Além disso, objetivou-se mensurar a pressão média do canal epidural antes e após a realização da técnica.

\section{MATERIAL E MÉTODOS}

O estudo foi aprovado pelo Comitê de Ética em Experimentação Animal (Cetea) da instituição de origem, sob protocolo número 1.42.13. Foram utilizados 18 caninos, machos, adultos, com peso médio de 12,9 $( \pm 3,6) \mathrm{kg}$, comprovadamente hígidos após realização de exame físico, hemograma, perfil bioquímico renal, hepático, e classificados pela American Society of Anesthesiologists (ASA) como ASA I. Os cães foram encaminhados para o procedimento de orquiectomia eletiva.

O internamento ocorreu no dia anterior ao procedimento, sendo os pacientes submetidos a jejum de sólidos de 10 horas e hídrico de seis horas. No dia do estudo, foram pré- medicados com acepromazina, na dose de $0,03 \mathrm{mg} / \mathrm{kg}$, e metadona, na dose de $0,3 \mathrm{mg} / \mathrm{kg}$, pela via intramuscular. Após 15 minutos, foram submetidos à cateterização da veia cefálica para inserção de um cateter calibre $22 \mathrm{G}$ para fluidoterapia, na taxa de $10 \mathrm{~mL} / \mathrm{kg} / \mathrm{h}$, e subsequente sedação com propofol, na dose de $2 \mathrm{mg} / \mathrm{kg}$, sem que fosse perdido o reflexo de retirada do membro e do panículo paravertebral. Ainda, foi realizada a tricotomia do dorso do animal, bem como a identificação e demarcação das vértebras lombares e torácicas para avaliação da progressão do bloqueio (Fig. 1). 


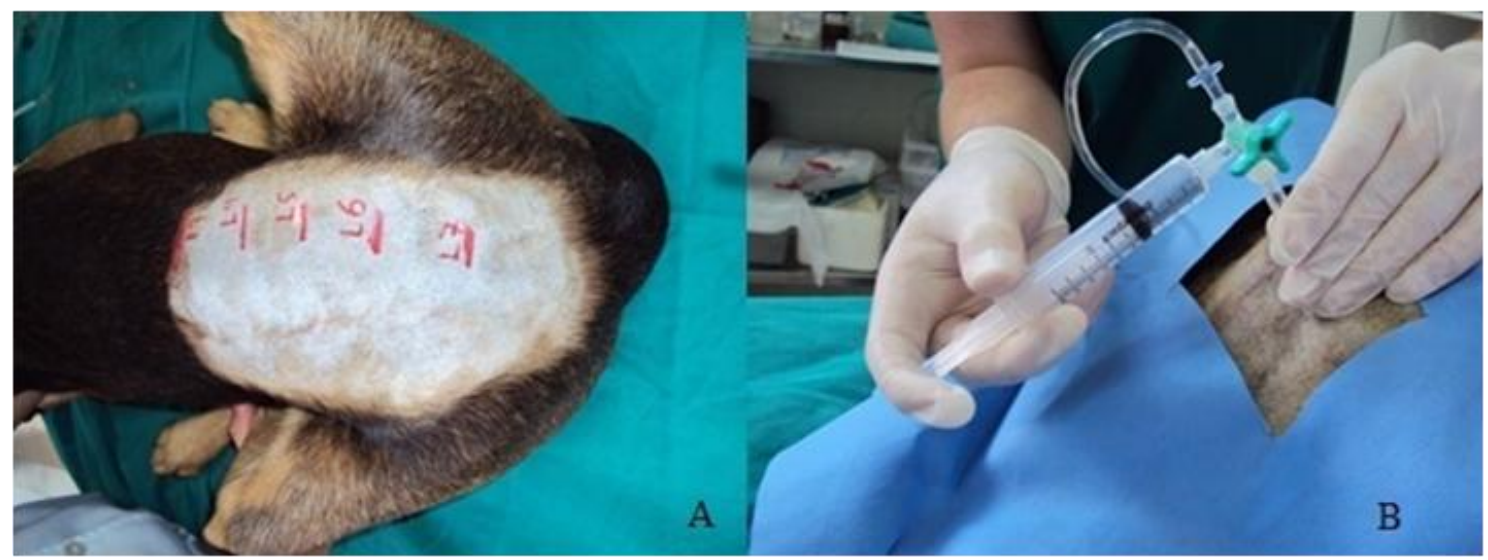

Figura 1. (A) Demarcação dos espaços intervertebrais em cães submetidos à anestesia epidural com lidocaína, bupivacaína ou com a sua associação para a posterior avaliação da progressão cranial pelo espaço por meio do pinçamento do panículo paravertebral. (B) Administração do tratamento pela via epidural e acoplamento ao canal de pressão para aferição da pressão do espaço epidural em cães. Notar nesta figura que a agulha foi introduzida já acoplada ao sistema de mensuração da pressão do espaço, não entrando, em nenhum momento, em contato com o ar ambiente.

Depois da sedação, os cães foram posicionados em decúbito esternal, com os membros pélvicos tracionados cranialmente, para a punção do espaço epidural com agulha espinhal 20G, após devida antissepsia, sendo essa agulha acoplada, antes mesmo de penetrar a pele do paciente, a um transdutor de pressão para mensuração da pressão média no canal epidural antes e após a administração dos tratamentos, o que não permitia que, ao atingir o espaço epidural, este tivesse contato com o ar ambiente (Fig. 1). A anestesia epidural foi realizada por um anestesiologista experiente, que administrava os tratamentos após transpor o ligamento amarelo, bem como observava a ausência de resistência ao administrá-los. Dessa maneira, os animais foram alocados em três grupos $(n=6)$ : lidocaína $(\mathrm{GL})$, cujos animais receberam lidocaína sem vasoconstrictor a $2 \%$, no volume de $0,25 \mathrm{~mL} / \mathrm{kg}$ $(5 \mathrm{mg} / \mathrm{kg})$, por via epidural; bupivacaína $(\mathrm{GB})$, cujos animais receberam bupivacaína sem vasoconstrictor a $0,5 \%$, no mesmo volume e via que o grupo anterior $(1,25 \mathrm{mg} / \mathrm{kg})$; e grupo lidocaína associada à bupivacaína (GLB), cujos animais receberam a associação de 1:1 de lidocaína a $2 \%$ e bupivacaína a $0,5 \%$ sem vasoconstrictor, no volume de $0,25 \mathrm{~mL} / \mathrm{kg}(2,5 \mathrm{e}$ $0,625 \mathrm{mg} / \mathrm{kg}$ respectivamente), pela via epidural. O tempo de administração do tratamento por via epidural foi padronizado em 30 segundos.

A avaliação do tempo de latência (minutos) foi realizada por meio de pinçamento do panículo paravertebral no nível da sétima vértebra lombar (L7) e da membrana interdigital dos membros pélvicos direito e esquerdo, considerando o tempo de latência o tempo para a perda de ambos os reflexos. Após a avaliação da latência, os animais foram mantidos em decúbito esternal por um período total de 15 minutos, sendo avaliada a progressão do anestésico pelo espaço epidural, por meio do reflexo panicular das vértebras lombares a partir de L7. Ato contínuo, o animal foi posicionado em decúbito dorsal para realização de orquiectomia.

Os parâmetros clínicos avaliados foram: frequência cardíaca (FC), por meio da estetoscopia; frequência respiratória $(f)$, por meio da visualização do gradil costal; e pressão arterial sistólica (PAS), com o auxílio do Doppler vascular, na artéria palmar superficial, e do manômetro anaeroide, nos seguintes momentos: M0 (15 minutos após a realização da MPA), M5, M15, M30 e M60 (cinco, 15, 30 e 60 minutos após a administração epidural). Ainda foi avaliada a pressão média no canal epidural antes da administração dos tratamentos e imediatamente após, por um transdutor de pressão conectado a um monitor multiparamétrico e à agulha espinhal (Fig. 1).

No pós-operatório, avaliou-se o período hábil (minutos) do bloqueio, por meio do pinçamento da membrana interdigital a cada 15 minutos, mediante a administração dos tratamentos até o 
retorno do reflexo de retirada do membro, bem como o período para recuperação total (minutos), caracterizado pelo momento em que o animal deambulava normalmente.

A análise estatística foi realizada com o auxílio do software SigmaPlot 12.0, empregando-se One Way RM ANOVA, seguida do teste de Dunnett para avaliação dos parâmetros clínicos em relação ao momento basal (M0), e One Way ANOVA seguido do teste de Tukey para avaliação dos parâmetros entre grupos. Diferenças foram consideradas significativas quando $\mathrm{P} \leq 0,05$.

\section{RESULTADOS E DISCUSSÃO}

Não foram observadas diferenças entre momentos dentro de cada grupo e entre os grupos para a FC e a $f$ (Tab. 1). Adetunji et al. (2001) também não verificaram alterações nesses parâmetros, ao utilizarem os mesmos fármacos e com volumes maiores $(0,3 \mathrm{~mL} / \mathrm{kg})$. Houve uma redução na PAS de 12,3\%,11,5\%, 13,6\% e
$11,5 \%$ do M5 ao M60 em relação ao M0, respectivamente, no GL. No GLB, a PAS diminuiu no M30 17,4\% em relação ao M0. Essa diminuição, embora clinicamente insignificante (Tab. 1), pode ser creditada à lidocaína, fato esse também observado por Sakonju et al. (2011), que, após a administração de lidocaína $2 \%$ no volume de $0,2 \mathrm{~mL} / \mathrm{kg}$ pela via epidural, verificaram diminuição da PA em cães. Corroborando esses dados, Cassu et al. (2008), quando utilizaram um volume maior $(0,41 \mathrm{~mL} / \mathrm{kg})$, também observaram diminuição dos parâmetros cardiorrespiratórios, incluída a PAS, após a administração de lidocaína isolada ou associada ao fentanil por via epidural em cães. Indo de encontro a esses estudos, Cruz et al. (1997) e Adetunji et al. (2001) não observaram diminuição da PA em seus respectivos estudos, no entanto, em ambos as pesquisas, a formulação de lidocaína apresentava epinefrina, o que ressalta a hipótese de que a queda na PA após a epidural é mediada pela vasodilatação local (Valverde, 2008).

Tabela 1. Valores médios e desvio-padrão dos parâmetros de: frequência cardíaca (FC), respiratória $(f)$ e pressão arterial sistólica (PAS) em cães submetidos à anestesia epidural com $0,25 \mathrm{~mL} / \mathrm{kg}$ de lidocaína $2 \%$ sem vasoconstrictor (GL);0,25mL/kg de bupivacaína $0,5 \%$ (GB); ou associação de $0,25 \mathrm{~mL} / \mathrm{kg}$ de ambas em uma proporção de 1:1 (GLB), após 15 minutos da administração da medicação pré-anestésica (M0), cinco (M5), 15 (M15), 30 (M30) e 60 (M60) minutos após a administração dos tratamentos por via epidural

\begin{tabular}{lcccccc}
\hline & & M0 & M5 & M15 & M30 & M60 \\
\hline \multirow{3}{*}{ FC $(\mathrm{bpm})$} & GL & $82 \pm 18$ & $78 \pm 31$ & $76 \pm 24$ & $72 \pm 19$ & $72 \pm 19$ \\
& GB & $80 \pm 19$ & $80 \pm 12$ & $82 \pm 26$ & $82 \pm 39$ & $106 \pm 52$ \\
& GLB & $74 \pm 21$ & $64 \pm 10$ & $61 \pm 11$ & $63 \pm 13$ & $74 \pm 30$ \\
$f(\mathrm{mpm})$ & GL & $58 \pm 71$ & $22 \pm 9$ & $19 \pm 8$ & $14 \pm 3$ & $17 \pm 3$ \\
& GB & $60 \pm 52$ & $24 \pm 10$ & $20 \pm 5$ & $22 \pm 5$ & $39 \pm 32$ \\
& GLB & $41 \pm 23$ & $18 \pm 6$ & $12 \pm 2$ & $15 \pm 3$ & $19 \pm 5$ \\
PAS $(\mathrm{mmHg})$ & GL & $154 \pm 17$ & $135 \pm 13 \mathrm{~A}$ & $136 \pm 19 \mathrm{~A}$ & $133 \pm 13 \mathrm{~A}$ & $136 \pm 17^{\mathrm{a}}$ \\
& GB & $155 \pm 26$ & $136 \pm 26$ & $131 \pm 22$ & $139 \pm 32$ & $154 \pm 28$ \\
& GLB & $143 \pm 23$ & $146 \pm 33$ & $142 \pm 24$ & $118 \pm 12 \mathrm{~A}$ & $135 \pm 21$ \\
\hline
\end{tabular}

A: Diferença em relação ao M0 após One Way RM ANOVA, seguido por Dunnet $(\mathrm{P} \leq 0,05)$.

Ao se analisar a progressão cranial do bloqueio anestésico após 15 minutos da sua administração pela via epidural (Tab. 2), percebeu-se que houve maior progressão do GL em relação aos demais grupos, em que $33,4 \% \quad(2 / 6)$ dos animais apresentaram bloqueio cranial a L2; enquanto isso, no GB e no GLB, uma grande porcentagem dos bloqueios, $88,3 \%$ e $66,8 \%$, respectivamente, não ultrapassaram L4. Rocha e Massone (2006) estudaram o segmento lombar de cães e demonstraram que o plexo nervoso simpático emerge de L3, sugerindo que bloqueios anestésicos nessa altura poderiam produzir efeitos simpaticolíticos, como o que foi observado no GL e no GLB. Os autores chamam a atenção para os dados individuais do paciente do GLB, que atingiu L3-L2 e, juntamente com a progressão mais cranial, foi também o que teve a menor PAS do grupo $(110 \mathrm{mmHg})$. Dessa forma, além de os anestésicos locais promoverem uma hipotensão pela vasodilatação local, discutida no parágrafo anterior, pode-se crer que, de maneira 
sinérgica, eles podem também progredir mais cranialmente, produzindo, então, efeitos simpaticolíticos. Os autores ressaltam que, apesar da queda significativa, em termos estatísticos da PAS, esses valores permaneceram acima dos valores de referência para a espécie canina. No entanto, tais achados foram verificados em cães conscientes, não se podendo inferir sobre possíveis alterações na PA causadas pela epidural em cães anestesiados.

Tabela 2. Altura do bloqueio a partir da sétima vértebra lombar (L7) e dos membros pélvicos (MP), verificada por meio do pinçamento interdigital e do panículo paravertebral, em cães, após 15 minutos de administração por via epidural, com $0,25 \mathrm{~mL} / \mathrm{kg}$ de lidocaína $2 \%$ sem vasoconstrictor (GL); $0,25 \mathrm{~mL} / \mathrm{kg}$ de bupivacaína $0,5 \%$ (GB); ou associação de $0,25 \mathrm{~mL} / \mathrm{kg}$ de ambas em uma proporção de 1:1 (GLB)

\begin{tabular}{lccc}
\multicolumn{1}{c}{ Altura do bloqueio } & GL & GB & GLB \\
\hline MP e L7 & - & - & $1 / 6(16,7 \%)$ \\
L7-L6 & $1 / 6(16,7 \%)$ & $1 / 6(16,7 \%)$ & - \\
L6-L5 & - & $1 / 6(16,7 \%)$ & $1 / 6(16,7 \%)$ \\
L5-L4 & $1 / 6(16,7 \%)$ & $3 / 6(50 \%)$ & $2 / 6(33,4 \%)$ \\
L4-L3 & $2 / 6(33,4 \%)$ & $1 / 6(16,7 \%)$ & $1 / 6(16,7 \%)$ \\
L3-L2 & - & - & $1 / 6(16,7 \%)$ \\
L2-L1 & $1 / 6(16,7 \%)$ & - & - \\
L1-T13 & $1 / 6(16,7 \%)$ & - & - \\
\hline
\end{tabular}

A pressão média no espaço epidural foi de $-1,5$ $( \pm 3,9) \mathrm{mmHg}$ e $41( \pm 16) \mathrm{mmHg}$ antes e após a administração dos tratamentos. Pouco mais da metade dos pacientes apresentou pressão negativa ao se atingir o espaço epidural $(55,5 \%)$. Iff e Moens (2010), ao avaliarem a pressão média do canal epidural, obtiveram como resultado uma pressão de $3( \pm 7,5) \mathrm{mmHg}$ e 35 $( \pm 21) \mathrm{mmHg}$ antes e após a administração dos tratamentos, respectivamente, em cães. Comparando-se o presente estudo com o de Iff e Moens (2010), parece lógico concluir que nem todos os animais apresentam pressão negativa no canal epidural, logo o teste da gota pendente pode não ser eficiente para localização do espaço epidural em todos os animais, tendo em vista que, em alguns animais, a agulha estará posicionada de maneira correta no espaço epidural, entretanto, no teste da gota pendente, esse animal apresentará um resultado falso negativo. Os resultados apresentados por Iff e Moens (2010) com relação à pressão do canal epidural são maiores que os do presente estudo devido à administração de solução salina no espaço epidural com o intuito de confirmar o posicionamento pela perda de resistência à injeção, sendo a mensuração realizada após essa administração, o que justifica os valores maiores encontrados no estudo de Iff e Moens (2010) quando comparado ao presente estudo.

Não foi observada diferença entre os grupos em relação ao período de latência dos fármacos (Tab. 3). Resultados similares foram descritos por Lizarraga et al. (2012), no bloqueio de metacarpo em ovinos, e por Li et al. (2013), na cauda de ratos, ao compararem a latência da lidocaína com a bupivacaína. Esses resultados vão ao encontro dos verificados por Seow et al. (1982), que analisaram a associação de lidocaína $2 \%$ com bupivacaína $0,5 \%$, em diferentes proporções, ou o uso desses fármacos, de maneira isolada, no bloqueio epidural em humanos, sendo observados tempos de latência similares entre os anestésicos locais isoladamente e entre as diferentes proporções de associação dos anestésicos locais. A latência dos anestésicos locais está relacionada ao pKa dos fármacos. Embora exista uma diferença matemática entre o pKa da lidocaína $(7,6)$ e o da bupivacaína $(8,1)$, essa pequena diferença parece não repercutir na rotina clínica. Relacionada a essa informação, observa-se uma elevada variabilidade no que tange à latência, levando os autores a crer que fatores teciduais individuais, e não somente o $\mathrm{pKa}$, exercem interferência na latência dos fármacos pela via epidural. Apenas Cruz et al. (1997) verificaram diferenças na latência entre os fármacos, nas mesmas doses do presente estudo, porém os fármacos apresentavam, em sua formulação, vasoconstrictor, o que justifica essas diferenças encontradas no trabalho de Cruz et al. (1997) e no presente estudo.

Quanto à duração do bloqueio (período hábil) e à recuperação total, essas foram menores no GL em relação ao GB (Tab. 3). O GLB obteve 
tempos intermediários entre o GL e o GB sem diferença significativa em relação aos demais grupos. Cruz et al. (1997), em cães, e Lawal e Adetunji (2009), em felinos, encontraram resultados semelhantes aos do presente estudo, demonstrando que a associação de lidocaína e bupivacaína apresenta duração de efeito intermediário quando comparada ao uso isolado dos fármacos. Os resultados de Adetunji et al. (2001) são os únicos na literatura em que a duração de ação da associação supera o efeito da bupivacaína isolada. Entretanto, os autores utilizaram, em seu estudo, as formulações de lidocaína com epinefrina e de bupivacaina sem epinefrina, logo, na associação, a adição de vasoconstrictor resultou em um prolongado efeito da bupivacaína, o que poderia justificar os resultados encontrados. Ribotzky et al. (1996) defendem que, ao se associarem dois fármacos com características químicas distintas, ocorrerá comprometimento da ação esperada do fármaco. Dessa forma, parece lógico pensar que, ao se associar a lidocaína com a bupivacaína na proporção de $1: 1$, ocorrerão $50 \%$ da atividade esperada de cada fármaco, ficando claro que a duração da associação deva compreender o intermédio da duração de cada fármaco isolado. Com isso, deve-se ter em mente que, ao se associarem anestésicos locais distintos, não se sabe o quanto se está afetando as suas características físico-químicas e, consequentemente, não se sabe ao certo o que esperar da associação. Portanto, os autores frisam que o anestésico local deve ser escolhido conforme o tempo e o planejamento esperados para cada procedimento cirúrgico, optando-se pela lidocaína quando se deseja uma ação mais curta, ou pela bupivacaína ao se almejar uma duração mais prolongada.

Tabela 3. Valores médios e desvio-padrão (em minutos) do período de latência, do período hábil e de recuperação total após anestesia epidural em cães com $0,25 \mathrm{~mL} / \mathrm{kg}$ de lidocaína $2 \%$ sem vasoconstrictor (GL); 0,25mL/kg de bupivacaína 0,5\% (GB); ou associação de 0,25mL/kg de ambas em uma proporção de $1: 1(\mathrm{GLB})$

\begin{tabular}{lccc}
\hline & GL & GB & GLB \\
\hline Latência (min) & $3,5 \pm 1,6$ & $4,5 \pm 4,4$ & $2,38 \pm 1,06$ \\
Período hábil (min) & $125 \pm 24 \mathrm{a}$ & $176 \pm 24 \mathrm{~b}$ & $153 \pm 35 \mathrm{ab}$ \\
Recuperação total (min) & $182 \pm 38 \mathrm{a}$ & $312 \pm 76 \mathrm{~b}$ & $257 \pm 45 \mathrm{ab}$ \\
\hline
\end{tabular}

Letras minúsculas: Diferença entre grupos após ANOVA seguido por Tukey $(\mathrm{P} \leq 0,05)$.

\section{CONCLUSÃO}

Com base na metodologia proposta, conclui-se que não há vantagens em associar a bupivacaína com a lidocaína, devendo-se optar entre ambas quando se deseja um efeito mais prolongado ou mais curto. Além disso, a lidocaína apresentou uma progressão mais cranial que a bupivacaína e a associação, reduzindo a PAS. Por fim, somente $55 \%$ dos pacientes apresentaram pressão negativa ao se atingir o espaço epidural, sendo o teste da gota pendente ineficaz em alguns casos.

\section{REFERÊNCIAS}

ADETUNJI, A.; AJADI, R.A.; ALADESAWE, T.A. Comparison of epidural anaesthesia with lignocaine, bupivacaine and lignocaine/ bupivacaine mixture in dogs. Isr. J. Vet. Med., v.56, p.85-89, 2001.
BERNARDS, C.M.; HILL, H.F. Physical and chemical properties of drug molecules governing their diffusion through the spinal meninges. Anesthesiology, v.77, p.750-756, 1992.

BUTTERWORTH, J.F.; STRICHARTZ, G.R. Molecular mechanisms of local anesthesia: a review. Anesthesiology, v.72, p.711-734, 1990.

CASSU, R.N.; STEVANIN, H.; KANASHIRO, C. et al. Anestesia epidural com lidocaína isolada e associada ao fentanil para realização de ováriossalpingo-histerectomia em cadelas. Arq. Bras. Med. Vet. Zootec., v.60, p.825-831, 2008.

CRUZ, M.L.; LUNA, S.P.L.; CLARK, R.M.O. et al. Epidural anaesthesia using lignocaine, bupivacaine or a mixture of lignocaine and bupivacaine in dogs. J. Vet. Anaesth., v.24, p.3032, 1997.

IFF, I.; MOENS, Y.P.S. Evaluation of extradural pressure waves and the 'lack of resistance' test to confirm extradural needle placement in dogs. Vet. J., v.185, p.328-331, 2010. 
LAWAL, F.M.; ADETUNJI, A.A comparison of epidural anaesthesia with lignocaine, bupivacaine and a lignocaine-bupivacaine mixture in cats. J. S. Afr. Vet. Assoc., v.80, p.243-246, 2009.

LI, Z.; CHAI, Y.; GONG, C. et al. Evaluation of the antinociceptive effects of lidocaine and bupivacaine on the tail nerves of healthy rats. Basic Clin. Pharmacol. Toxicol., v.113, p.31-36, 2013.

LIZARRAGA, I.; JANOVYAK, E.; BETHS, T. Comparing lidocaine, bupivacaine and a lidocaine-bupivacaine mixture as a metacarpal block in sheep. Vet. J., v.197, p.515-518, 2012.

RIBOTSKY, B.M.; BERKOWITZ, K.D.; MONTAGUE, J.R. Local anesthetics: is there an advantage to mixing solutions? J. Am. Podiatr. Med. Assoc., v.86, p.487-491, 1996.
ROCHA, L.M.S.; MASSONE F. Estudo anatomo-anestesiológico do segmento lombar (L1 a L6) em cães. Braz. J. Vet. Res. Anim. Sci., v.43, p.167-177, 2006.

SAKONJU, I.; MAEDA, K.; KARASAWA, K. et al. Plasma concentration and cardiovascular effects of lidocaine during continuous epidural administration in dogs anesthetized with isoflurane. J. Vet. Med. Sci., v.73, p.393-398, 2011.

SEOW, L.T.; LIPS, F.J.; COUSINS, M.J. et al. Lidocaine and bupivacaine mixtures for epidural blockade. Anesthesiology, v.53, p.177-183, 1982.

VALVERDE, A. Epidural analgesia and anesthesia in dogs and cats. Vet. Clin. N. Am. Small Anim. Pract., v.38, p.1205-1231, 2008. 